\title{
Upper bounds for the decay rate in a nonlocal $p$-Laplacian evolution problem
}

\author{
Carlos Esteve1, Julio D Rossi ${ }^{1,2}$ and Angel San Antolin ${ }^{1 *}$
}

\section{"Correspondence:}

angel.sanantolin@ua.es

'Departamento de Análisis

Matemático, Universidad de

Alicante, Ap. correos 99, Alicante 03080, Spain

Full list of author information is available at the end of the article

\begin{abstract}
We obtain upper bounds for the decay rate for solutions to the nonlocal problem $\partial_{t} u(x, t)=\int_{\mathbb{R}^{n}} J(x, y)|u(y, t)-u(x, t)|^{p-2}(u(y, t)-u(x, t)) d y$ with an initial condition $u_{0} \in L^{1}\left(\mathbb{R}^{n}\right) \cap L^{\infty}\left(\mathbb{R}^{n}\right)$ and a fixed $p>2$. We assume that the kernel $J$ is symmetric, bounded (and therefore there is no regularizing effect) but with polynomial tails, that is, we assume a lower bounds of the form $J(x, y) \geq c_{1}|x-y|^{-(n+2 \sigma)}$, for $|x-y|>c_{2}$ and $J(x, y) \geq c_{1}$, for $|x-y| \leq c_{2}$. We prove that $\|u(\cdot, t)\|_{L q\left(\mathbb{R}^{n}\right)} \leq C t^{-\frac{n}{(p-2) n+2 \sigma}}{ }^{\left(1-\frac{1}{q}\right)}$ for $q \geq 1$ and $t$ large.

MSC: $35 \mathrm{~K} 05 ; 45 \mathrm{P0}$; 35B40
\end{abstract}

Keywords: nonlocal diffusion; decay rates

\section{Introduction}

In this paper we deal with nonlocal Cauchy problems of the form

$$
\partial_{t} u(x, t)=\int_{\mathbb{R}^{n}} J(x, y)|u(y, t)-u(x, t)|^{p-2}(u(y, t)-u(x, t)) d y
$$

for $t \in \mathbb{R}_{+}$and $x \in \mathbb{R}^{n}$ with $n \geq 2$, a fixed $p>2$ and an initial condition $u(x, 0)=u_{0}(x)$ satisfying $u_{0} \in L^{1}\left(\mathbb{R}^{n}\right) \cap L^{\infty}\left(\mathbb{R}^{n}\right)$. As regards the kernel $J$, we will always assume that it is a bounded and symmetric function defined for $(x, y) \in \mathbb{R}^{n} \times \mathbb{R}^{n}$ together with the integrability condition $J(\cdot, y) \in L^{1}\left(\mathbb{R}^{n}\right)$ for all $y \in \mathbb{R}^{n}$. Under these hypotheses existence and uniqueness of a solution follow from a fixed point argument as in [1].

Nonlocal problems have been recently widely used to model diffusion processes (see [2] and [3] for a general nonlocal vector calculus). Problem (1.1) and its stationary version have been considered recently in connection with real applications, for example to peridynamics or a recent model for elasticity. We quote for instance [4-8] and the recent book [1].

Our main goal here is to obtain upper bounds for the asymptotic behavior of the solution of (1.1) as $t \rightarrow+\infty$. It is expected that the diffusive nature of the equation implies that the solution goes to zero when $t \rightarrow+\infty$.

To obtain our results the key assumptions are the following lower bounds for $J$ :

$$
\begin{aligned}
& J(x, y) \geq c_{1}|x-y|^{-(n+2 \sigma)} \quad \text { for }|x-y|>c_{2} \quad \text { and } \\
& J(x, y) \geq c_{1} \quad \text { for }|x-y| \leq c_{2}
\end{aligned}
$$

for certain constants $c_{1}, c_{2}>0$, and $\sigma \in(0,1)$. For simplicity we will assume $c_{2}=1$.

\section{Springer}

(02014 Esteve et al.; licensee Springer. This is an Open Access article distributed under the terms of the Creative Commons Attribution License (http://creativecommons.org/licenses/by/2.0), which permits unrestricted use, distribution, and reproduction in any medium, provided the original work is properly cited. 
The main result of this paper reads as follows.

Theorem 1.1 Let $n \geq 2, q \in[1,+\infty)$ and $\sigma \in(0,1)$. Let $J$ be a kernel satisfying (1.2). Then the solution of (1.1) associated to an initial condition $u_{0} \in L^{1}\left(\mathbb{R}^{n}\right) \cap L^{\infty}\left(\mathbb{R}^{n}\right)$ decays in $L^{q}\left(\mathbb{R}^{n}\right)$ with the upper bound

$$
\|u(\cdot, t)\|_{L^{q\left(\mathbb{R}^{n}\right)}} \leq C t^{-\frac{n}{(p-2) n+2 \sigma}\left(1-\frac{1}{q}\right)}
$$

where the constant $C$ depends on $u_{0}, q, \sigma$, and $n$.

Let us end the introduction with some comments on the previous bibliography. For the linear case, $p=2$, and for smooth kernels $J$ with compact support, it is proven in [9] that the solution $u$ of (1.1) has the decay estimate

$$
\|u(\cdot, t)\|_{L^{q}\left(\mathbb{R}^{n}\right)} \leq C t^{-\frac{n}{2}\left(1-\frac{1}{q}\right)}
$$

for any $q \in[1, \infty)$. Note that this decay rate is the same as the one that holds for solutions of the classical Heat equation. In the case of an equation in convolution form, that is, when $J(x, y)=K(x-y)$ with $K$ a nonnegative radial function, not necessarily compactly supported, it is proven in [10] that the solutions of equations with the form (1.1) have the decay estimate

$$
\|u(\cdot, t)\|_{L^{q\left(\mathbb{R}^{n}\right)}} \leq C t^{-\frac{n}{2 \sigma}\left(1-\frac{1}{q}\right)}
$$

provided the function $K$ has a Fourier transform satisfying the expansion $\hat{K}(\xi)=1-$ $A|\xi|^{2 \sigma}+o\left(|\xi|^{2 \sigma}\right)$ for $\xi \sim 0$, where $A>0$ is a constant. In this case the decay estimate is analogous to the one for the $\sigma$-order fractional heat equation, $v_{t}=-(-\Delta)^{\sigma} v$, with $\sigma \in(0,1)$. We also note that the convolution form of the equation allows the use of Fourier analysis to obtain this result. However, the use of Fourier analysis is not helpful here due to fact that our operator is not in convolution form. In spite of this difficulty, energy methods can be applied; see $[9,11]$. We also mention the recent reference [12], where similar estimates can be found for nonlocal equations with unbounded kernels. We borrow ideas and techniques from these references. In particular we use Proposition 3.2 of [11] (whose proof is included here for completeness). However, we have to point out that in [12] and [11] only the linear case, that is, $p=2$, was treated, while here we deal with (1.1) for any $p \geq 2$. For examples of kernels with exponential decay bounds we refer to [13] and [14]. Finally, we point out that our results are also valid for unbounded kernels (in the spirit of [12]) since only lower bounds are assumed in (1.2).

The case $1 \leq p<2$ remains open as well as the corresponding estimate for the $L^{\infty}$-norm.

\section{Basic facts and preliminaries}

First, we need to introduce fractional Sobolev spaces and their seminorms, we refer to [15] for details. For $\sigma \in(0,1)$ and $r \in[1, \infty), W^{\sigma, r}\left(\mathbb{R}^{n}\right)$ is the fractional Sobolev space of all $L^{r}\left(\mathbb{R}^{n}\right)$ functions with finite fractional seminorm $[v]_{\sigma, r}$, given by

$$
[v]_{\sigma, r}^{r}=\iint_{\mathbb{R}^{2 n}} \frac{|v(x+z)-v(x)|^{r}}{|z|^{n+r \sigma}} d x d z .
$$


Under these definitions, we have the following fractional Sobolev-type inequality: there exists a constant $C>0$ such that, for each $v \in W^{\sigma, r}\left(\mathbb{R}^{n}\right)$ with $\sigma r<n$, we have

$$
\|v\|_{L^{s}\left(\mathbb{R}^{n}\right)}^{r} \leq C[v]_{\sigma, r}^{r}
$$

where $s=n r /(n-\sigma r)$ (see [15]).

First, we consider a positive smooth function $\psi: \mathbb{R}^{n} \rightarrow \mathbb{R}$ with the following properties:

$$
\operatorname{supp}(\psi) \subset B_{1} \quad \text { and } \quad \int_{\mathbb{R}^{n}} \psi(x) d x=1 .
$$

With the aid of this function, we split a function $u$ into two parts. We will denote the 'smooth' part of $u$ as $v$ and the remaining as $w$. We let

$$
v(x, t):=\int_{\mathbb{R}^{n}} \psi(x-z) u(z, t) d z ; \quad w(x, t):=u(x, t)-v(x, t) .
$$

Sometimes, for simplicity in the notation and where the context is clear, we will write $u$, $v$, and $w$ as functions depending only of $x$.

As a first property of this decomposition we find that each $L^{r}$ norm of the functions $v$ and $w$ is controlled by the corresponding norm of $u$.

Lemma 2.1 Let $v$ and $w$ be given by (2.4). For each $r \in(1,+\infty)$, we have

$$
\|v\|_{L^{r}\left(\mathbb{R}^{n}\right)} \leq\|u\|_{L^{r}\left(\mathbb{R}^{n}\right)} \quad \text { and } \quad\|w\|_{L^{r}\left(\mathbb{R}^{n}\right)} \leq\|u\|_{L^{r}\left(\mathbb{R}^{n}\right)} .
$$

Proof We start with $v$. Denoting $r^{\prime}=r /(r-1)$ the Hölder conjugate of $r$ and using the definition of $v$, we have

$$
\begin{aligned}
\int_{\mathbb{R}^{n}}|v(x)|^{r} d x & =\int_{\mathbb{R}^{n}}\left|\int_{\mathbb{R}^{n}} \psi(x-y) u(y) d y\right|^{r} d x \\
& =\int_{\mathbb{R}^{n}}\left|\int_{\mathbb{R}^{n}} \psi(x-y)^{1 / r^{\prime}} \psi(x-y)^{1 / r} u(y) d y\right|^{r} d x \\
& \leq \int_{\mathbb{R}^{n}}\left[\left(\int_{\mathbb{R}^{n}} \psi(x-y) d y\right)^{1 / r^{\prime}}\left(\int_{\mathbb{R}^{n}} \psi(x-y)|u(y)|^{r} d y\right)^{1 / r}\right]^{r} d x \\
& =\int_{\mathbb{R}^{n}}|u(y)|^{r} \int_{\mathbb{R}^{n}} \psi(x-y) d x d y \\
& \leq \int_{\mathbb{R}^{n}}|u(y)|^{r} d y .
\end{aligned}
$$

The inequality for $w$ easily follows immediately from the triangular inequality in $L^{r}$.

Now we state a key result to get the desired estimate on the decay rate.

Proposition 2.2 Let $n \geq 2$ and let $J: \mathbb{R}^{n} \times \mathbb{R}^{n} \rightarrow \mathbb{R}_{+}$be a kernel satisfying (1.2), $\psi$ satisfying (2.3), $\beta \in[\sigma, 1)$, and $r>\max \{1,2 \beta\}$. Then there exists a constant $C>0$ such that, for all $u \in L^{r}\left(\mathbb{R}^{n}\right)$ and $v, w$ defined in $(2.4)$, we have

$$
[v]_{2 \beta r^{-1, r}}^{r}+\|w\|_{L^{r}\left(\mathbb{R}^{n}\right)}^{r} \leq C \iint_{\mathbb{R}^{2 n}} J(x, y)|u(x)-u(y)|^{r} d x d y .
$$

The constant $C$ depends on $\psi, \beta, r$, and $n$. 
Proof For the estimate concerning $w$, we have

$$
\begin{aligned}
\int_{\mathbb{R}^{n}}|w(x)|^{r} d x & =\int_{\mathbb{R}^{n}}|u(x)-v(x)|^{r} d x \\
& =\int_{\mathbb{R}^{n}}\left|u(x)-\int_{\mathbb{R}^{n}} \psi(x-z) u(z) d z\right|^{r} d x \\
& =\int_{\mathbb{R}^{n}}\left|\int_{\mathbb{R}^{n}} \psi(x-z)(u(x)-u(z)) d z\right|^{r} d x \\
& =\int_{\mathbb{R}^{n}}\left|\int_{\mathbb{R}^{n}} \psi(x-z)^{1 / r^{\prime}} \psi(x-z)^{1 / r}(u(x)-u(z)) d z\right|^{r} d x .
\end{aligned}
$$

Applying Hölder's inequality, we get

$$
\begin{aligned}
\int_{\mathbb{R}^{n}}|w(x)|^{r} d x & \leq \int_{\mathbb{R}^{n}}\left(\int_{\mathbb{R}^{n}} \psi(x-z) d z\right)^{r / r^{\prime}}\left(\int_{\mathbb{R}^{n}} \psi(x-z)|u(x)-u(z)|^{r} d z\right) d x \\
& \leq \int_{\mathbb{R}^{n}} \int_{\mathbb{R}^{n}} \psi(x-z)|u(x)-u(z)|^{r} d z d x,
\end{aligned}
$$

where $r^{\prime}=r /(r-1)$.

Since $\psi$ is supported in $B_{1}$, we have $\psi(x-z) \leq J(x, z)$ for all $|x-z| \geq 1$, and, since $J$ verifies $J(x, z) \geq c_{1}$ for $|x-z| \leq 1$, there exists a constant $C$ depending only on $|\psi|_{\infty}$ such that $\psi(x-z) \leq C J(x, z)$. Then

$$
\|w\|_{L^{r}\left(\mathbb{R}^{n}\right)}^{r} \leq C \iint_{\mathbb{R}^{2 n}} J(x, y)|u(x)-u(y)|^{r} d x d y
$$

Now we deal with the term with $v$. We split the fractional seminorm as

$$
\begin{aligned}
{[v]_{2 \beta r^{-1}, r}^{r} } & =\iint_{|x-y|>1} \frac{|v(x)-v(y)|^{r}}{|x-y|^{n+2 \beta}} d x d y+\iint_{|x-y| \leq 1} \frac{|v(x)-v(y)|^{r}}{|x-y|^{n+2 \beta}} d x d y \\
& =: I_{\text {ext }}+I_{\text {int }}
\end{aligned}
$$

and look at these integrals separately. For $I_{\text {ext }}$, using the definition of $v$ we have

$$
I_{\mathrm{ext}}=\iint_{|x-y|>1}\left|\int_{\mathbb{R}^{n}}(u(x-z)-u(y-z)) \psi(z) d z\right|^{r}|x-y|^{-(n+2 \beta)} d x d y .
$$

Now, we can think of the measure $\mu(d z)=\psi(z) d z$ as a probability measure (because of (2.3)), and since the function $t \mapsto|t|^{r}$ is convex in $\mathbb{R}$, we can apply Jensen's inequality on the $d z$-integral in right-hand side of the last expression to obtain

$$
I_{\mathrm{ext}} \leq \iint_{|x-y|>1} \int_{\mathbb{R}^{n}}|u(x-z)-u(y-z)|^{r} \psi(z) d z|x-y|^{-(n+2 \beta)} d x d y,
$$

which, after an application of Fubini's theorem, gives

$$
I_{\mathrm{ext}} \leq \int_{\mathbb{R}^{n}} \psi(z)\left(\iint_{|x-y|>1}|u(x-z)-u(y-z)|^{r}|x-y|^{-(n+2 \beta)} d x d y\right) d z
$$


Then, applying the change $\tilde{x}=x-z, \tilde{y}=y-z$ in the $d x d y$ integral and using (2.3), we conclude

$$
\begin{aligned}
I_{\mathrm{ext}} & \leq \int_{\mathbb{R}^{n}} \psi(z)\left(\iint_{|\tilde{x}-\tilde{y}|>1}|u(\tilde{x})-u(\tilde{y})|^{r}|\tilde{x}-\tilde{y}|^{-(n+2 \beta)} d \tilde{x} d \tilde{y}\right) d z \\
& =\iint_{|\tilde{x}-\tilde{y}|>1}|u(\tilde{x})-u(\tilde{y})|^{r}|\tilde{x}-\tilde{y}|^{-(n+2 \beta)} d \tilde{x} d \tilde{y} .
\end{aligned}
$$

Using this last expression, we obtain from the assumption (1.2) that

$$
I_{\mathrm{ext}} \leq C \iint_{\mathbb{R}^{2 n}} J(x, y)|u(x)-u(y)|^{r} d x d y
$$

Now we deal with $I_{\text {int }}$. In this case, using the definition of $v$, we can write

$$
I_{\mathrm{int}}=\iint_{|x-y|<1}\left|\int_{\mathbb{R}^{n}} u(z)(\psi(x-z)-\psi(y-z)) d z\right|^{r}|x-y|^{-(n+2 \beta)} d x d y .
$$

Note that by using (2.3), we have for all $x, y \in \mathbb{R}^{n}$

$$
\int_{\mathbb{R}^{n}} u(x)(\psi(x-z)-\psi(y-z)) d z=u(x)\left(\int_{\mathbb{R}^{n}} \psi(x-z) d z-\int_{\mathbb{R}^{n}} \psi(y-z) d z\right)=0,
$$

and then

$$
\int_{\mathbb{R}^{n}} u(z)(\psi(x-z)-\psi(y-z)) d z=\int_{\mathbb{R}^{n}}(u(z)-u(x))(\psi(x-z)-\psi(y-z)) d z .
$$

Thus, using this equality with (2.7), we get

$$
I_{\mathrm{int}}=\iint_{|x-y|<1}\left|\int_{\mathbb{R}^{n}}(u(z)-u(x))(\psi(x-z)-\psi(y-z)) d z\right|^{r}|x-y|^{-(n+2 \beta)} d x d y .
$$

However, note that if $|x-z| \geq 2$ in the $d z$ integral, since $|x-y|<1$ necessarily $|y-z|>1$. Then, due to the fact that $\psi$ is supported in the unit ball, the contribution of the integrand when $|x-z| \geq 2$ is null in the $d z$ integral. Taking this into account, applying Hölder's inequality into the $d z$-integral, we have

$$
\begin{aligned}
I_{\text {int }}= & \iint_{|x-y|<1}\left|\int_{|x-z|<2}(u(z)-u(x))(\psi(x-z)-\psi(y-z)) d z\right|^{r} \\
& \times|x-y|^{-(n+2 \beta)} d x d y \\
\leq & \iint_{|x-y|<1}\left(\int_{|x-z|<2}|u(z)-u(x)|^{r} d z\right) \\
& \times\left(\int_{|x-\tilde{z}|<2}|\psi(x-\tilde{z})-\psi(y-\tilde{z})|^{r^{\prime}} d \tilde{z}\right)^{r / r^{\prime}}|x-y|^{-(n+2 \beta)} d x d y .
\end{aligned}
$$

By Fubini's theorem we can write

$$
I_{\text {int }}=\int_{x \in \mathbb{R}^{n}}\left(\int_{|x-z|<2}(u(z)-u(x))^{r} d z\right) \Psi(x) d x
$$


where

$$
\Psi(x)=\int_{|x-y|<1}\left(\int_{|x-\tilde{z}|<2}|\psi(x-\tilde{z})-\psi(y-\tilde{z})|^{r^{\prime}} d \tilde{z}\right)^{r / r^{\prime}}|x-y|^{-(n+2 \beta)} d y .
$$

Using the regularity of $\psi$, we have

$$
\begin{aligned}
\Psi(x) & \leq \int_{|x-y|<1}\left(\int_{|x-\tilde{z}|<2}\|D \psi\|_{\infty}^{r^{\prime}}|x-y|^{r^{\prime}} d \tilde{z}\right)^{r / r^{\prime}}|x-y|^{-(n+2 \beta)} d y \\
& \leq\|D \psi\|_{\infty}^{r}\left|B_{2}\right|^{r / r^{\prime}} \int_{|x-y|<1}|x-y|^{r}|x-y|^{-(n+2 \beta)} d y,
\end{aligned}
$$

and since $r>2 \beta$, we conclude that the last integral is convergent, obtaining

$$
\Psi(x) \leq C_{n, \beta, r}\|D \psi\|_{\infty}^{r}\left|B_{2}\right|^{r / r^{\prime}},
$$

which leads to the following estimate for $I_{\text {int }}$ :

$$
I_{\text {int }} \leq C \int_{x \in \mathbb{R}^{n}} \int_{|x-z|<2}|u(z)-u(x)|^{r} d z d x .
$$

From this, it is easy to get

$$
I_{\text {int }} \leq C \int_{|x-z| \leq 2} \frac{|u(z)-u(x)|^{r}}{(1+|x-z|)^{n+2 \beta}} d z d x,
$$

which, by the use of (1.2), let us conclude that

$$
I_{\text {int }} \leq C \iint_{\mathbb{R}^{2 n}} J(x, y)|u(x)-u(y)|^{r} d x d y .
$$

This last estimate together with (2.6) concludes the proof.

\section{Proof of Theorem 1.1}

As mentioned in the introduction, existence and uniqueness of solutions to problem (1.1) follows as in [1]. In fact, the symmetry, boundedness, and integrability assumptions over $J$ allow us to perform a fixed point argument to obtain the following result, whose proof is omitted.

Theorem 3.1 Let $u_{0} \in L^{1}\left(\mathbb{R}^{n}\right) \cap L^{\infty}\left(\mathbb{R}^{n}\right)$, then there exists a unique solution $u \in C([0,+\infty)$, $\left.L^{1}\left(\mathbb{R}^{n}\right) \cap L^{\infty}\left(\mathbb{R}^{n}\right)\right)$ of equation (1.1). This solution satisfies $\|u(\cdot, t)\|_{L^{1}\left(\mathbb{R}^{n}\right)} \leq\left\|u_{0}\right\|_{L^{1}\left(\mathbb{R}^{n}\right)}$ and $\|u(\cdot, t)\|_{L^{\infty}\left(\mathbb{R}^{n}\right)} \leq\left\|u_{0}\right\|_{L^{\infty}\left(\mathbb{R}^{n}\right)}$ for all $t \geq 0$.

Now, let us introduce the main idea behind the energy methods. To clarify the exposition, let us perform these computations in the local case and next see how we can adapt them to our nonlocal problem with the help of Proposition 2.2. Let us describe briefly how the energy method can be applied to obtain decay estimates for local problems. Let us begin with the simpler case of the estimate for solutions to the $p$-Lapacian evolution equation in $L^{2}$-norm. Let $u$ be a solution to

$$
\partial_{t} u=\Delta_{p} u
$$


If we multiply the equation by $u$ and integrate in $\mathbb{R}^{n}$, we obtain

$$
\partial_{t} \int_{\mathbb{R}^{n}} \frac{1}{2} u^{2}(x, t) d x=-\int_{\mathbb{R}^{n}}|\nabla u(x, t)|^{p} d x
$$

Now we use Sobolev's inequality

$$
\int_{\mathbb{R}^{n}}|\nabla u(x, t)|^{p} d x \geq C\left(\int_{\mathbb{R}^{n}}|u(x, t)|^{p^{*}} d x\right)^{p / p^{*}}
$$

with $p^{*}=p n /(n-p)$ to obtain

$$
\partial_{t} \int_{\mathbb{R}^{n}} u^{2}(x, t) d x \leq-C\left(\int_{\mathbb{R}^{n}}|u(x, t)|^{p^{*}} d x\right)^{p / p^{*}} .
$$

If we use interpolation and that $\|u(\cdot, t)\|_{L^{1}\left(\mathbb{R}^{n}\right)} \leq C\left(u_{0}\right)$ for any $t>0$, we have

$$
\|u(\cdot, t)\|_{L^{2}\left(\mathbb{R}^{n}\right)} \leq\|u(\cdot, t)\|_{L^{1}\left(\mathbb{R}^{n}\right)}^{\alpha}\|u(\cdot, t)\|_{L^{p^{*}}\left(\mathbb{R}^{n}\right)}^{1-\alpha} \leq C\|u(\cdot, t)\|_{L^{p^{*}}\left(\mathbb{R}^{n}\right)}^{1-\alpha}
$$

with $\alpha$ determined by

$$
\frac{1}{2}=\alpha+\frac{1-\alpha}{p^{*}}, \quad \text { that is, } \alpha=\left(\frac{1}{2}-\frac{1}{p^{*}}\right) \frac{p^{*}}{\left(p^{*}-1\right)} .
$$

Hence we get

$$
\partial_{t} \int_{\mathbb{R}^{n}} u^{2}(x, t) d x \leq-C\left(\int_{\mathbb{R}^{n}} u^{2}(x, t) d x\right)^{\frac{1}{1-\alpha}}
$$

from where the decay estimate

$$
\|u(\cdot, t)\|_{L^{2}\left(\mathbb{R}^{n}\right)} \leq C t^{-\frac{1}{2}\left(\frac{n}{n(p-2)+2}\right)}, \quad t>0,
$$

follows. To obtain a decay bound for $\|u(\cdot, t)\|_{L^{q}\left(\mathbb{R}^{n}\right)}$ we can use the same idea multiplying by $u^{q-1}$ at the beginning.

Now we are ready to proceed with the proof of our main result.

Proof of Theorem 1.1 The symmetry assumption on $J$ allows us to mimic this idea and use an energy approach in order to get Theorem 1.1. Roughly speaking, this assumption allows us to 'integrate by parts' (1.1). For $q=1$ the proof is finished by Theorem 3.1. For $q>1$ we multiply the equation by $q|u|^{q-2} u$ and integrate, obtaining the identity

$$
\begin{aligned}
\partial_{t} \int_{\mathbb{R}^{n}}|u(x)|^{q} d x= & -\frac{q}{2} \iint_{\mathbb{R}^{2 n}} J(x, y)|u(y)-u(x)|^{p-2}(u(y)-u(x)) \\
& \times\left(|u(y)|^{q-2} u(y)-|u(x)|^{q-2} u(x)\right) d y d x,
\end{aligned}
$$

where we omitted the dependence on $t$ of the function $u$ for simplicity. 
Now we recall the following inequality (whose proof is straightforward): let $q>1$ and $a, b \neq 0$. Then there exists a constant $C$ depending only on $q$, such that

$$
(a-b)\left(|a|^{q-2} a-|b|^{q-2} b\right) \geq C|a-b|^{q} .
$$

Hence, using this inequality with (3.1), we conclude

$$
\partial_{t}\|u\|_{L^{q}\left(\mathbb{R}^{n}\right)}^{q} \leq-C \iint_{\mathbb{R}^{2 n}} J(x, y)|u(y)-u(x)|^{p-2+q} d y d x=:-C E(u) .
$$

Note that we find that the $L^{q}$-norm of $u$ is decreasing in $t$. At this point we would like to use Sobolev's inequality, which is not available due to the lack of regularizing effect of our nonlocal operator. Instead we will use Proposition 2.2, which involves a good control of the smooth part $v$ (but we have to take care of the rough part $w$ ).

Let us fix $q \geq 2$ (the case $1<q<2$ will be tackled at the end of this proof using interpolation). By the definition of $v$ and $w$ in (2.4), we have

$$
\|u\|_{L^{q}\left(\mathbb{R}^{n}\right)}^{q} \leq 2^{q-1}\left(\|v\|_{L^{q}\left(\mathbb{R}^{n}\right)}^{q}+\|w\|_{L^{q}\left(\mathbb{R}^{n}\right)}^{q}\right) .
$$

Now we note that $v$ belongs to $L^{p}$ for all $p$. Hence, we can interpolate, obtaining

$$
\|v\|_{L^{q\left(\mathbb{R}^{n}\right)}}^{q} \leq\|v\|_{L^{s}\left(\mathbb{R}^{n}\right)}^{q \theta}\|v\|_{L^{1}\left(\mathbb{R}^{n}\right)}^{q(1-\theta)}
$$

with

$$
s=\frac{n(p+q-2)}{n-2 \sigma},
$$

where $\theta$ is given by

$$
\frac{1}{q}=\frac{\theta}{s}+(1-\theta), \quad \text { that is, } \theta=\frac{s(q-1)}{q(s-1)} .
$$

Recalling $\|v\|_{L^{1}\left(\mathbb{R}^{n}\right)} \leq\|u(\cdot, t)\|_{L^{1}\left(\mathbb{R}^{n}\right)} \leq\left\|u_{0}\right\|_{L^{1}\left(\mathbb{R}^{n}\right)}$ and the Sobolev-type inequality (2.2), we obtain

$$
\|v\|_{L^{q}\left(\mathbb{R}^{n}\right)}^{q} \leq C[v]_{\tilde{\sigma}, p+q-2}^{q \theta},
$$

where $\tilde{\sigma}=2 \sigma(p+q-2)^{-1}$ and the constant $C$ depends on $u_{0}, q$, $\sigma$, and $n$.

Concerning $w$ we can also interpolate and obtain

$$
\|w\|_{L^{q}\left(\mathbb{R}^{n}\right)}^{q} \leq\|w\|_{L^{p+q-2}\left(\mathbb{R}^{n}\right)}^{q \gamma}\|w\|_{L^{1}\left(\mathbb{R}^{n}\right)}^{q(1-\gamma)}
$$

with $\gamma$ given by

$$
\frac{1}{q}=\frac{\gamma}{p+q-2}+(1-\gamma), \quad \text { that is, } \gamma=\frac{(p+q-2)(q-1)}{q(p+q-3)} .
$$

Note that we are using that $p>2$ here. Now we use

$$
\|w\|_{L^{1}\left(\mathbb{R}^{n}\right)} \leq\|u(\cdot, t)\|_{L^{1}\left(\mathbb{R}^{n}\right)} \leq\left\|u_{0}\right\|_{L^{1}\left(\mathbb{R}^{n}\right)}
$$


to get

$$
\|w\|_{L^{q}\left(\mathbb{R}^{n}\right)}^{q} \leq C\|w\|_{L^{p+q-2}\left(\mathbb{R}^{n}\right)}^{q \gamma}
$$

with $C$ depending on $u_{0}, q, \sigma$, and $n$.

From (3.3), (3.4), and (3.5) we obtain

$$
\|u\|_{L^{q}\left(\mathbb{R}^{n}\right)}^{q} \leq C[v]_{\tilde{\sigma}, p+q-2}^{q \theta}+C\|w\|_{L^{p+q-2}\left(\mathbb{R}^{n}\right)}^{q \gamma} .
$$

Now we use Proposition 2.2, with $r=p+q-2$ and $\beta=\sigma$, to obtain

$$
\|v\|_{L^{s}\left(\mathbb{R}^{n}\right)}^{q \theta} \leq C(E(u))^{\frac{q \theta}{p+q-2}} \quad \text { and } \quad\|w\|_{L^{p+q-2}\left(\mathbb{R}^{n}\right)}^{q \gamma} \leq C(E(u))^{\frac{q \gamma}{p+q-2}}
$$

and we conclude that

$$
\|u\|_{L^{q}\left(\mathbb{R}^{n}\right)}^{q} \leq C(E(u))^{\frac{q \theta}{p+q-2}}+C(E(u))^{\frac{q \gamma}{p+q-2}}
$$

that is,

$$
H^{-1}\left(\|u\|_{L^{q\left(\mathbb{R}^{n}\right)}}^{q}\right) \leq E(u)
$$

with $H(z)=C z^{\frac{q \theta}{p+q-2}}+C z^{\frac{q \gamma}{p+q-2}}$. Since $\|u\|_{L^{q}\left(\mathbb{R}^{n)}\right.}^{q}(t) \leq\left\|u_{0}\right\|_{L^{q}\left(\mathbb{R}^{n)}\right.}^{q}$ (recall that the $L^{q}$-norm of the solution decreases) and $\frac{q \theta}{p+q-2}<\frac{q \gamma}{p+q-2}$, we have

$$
H^{-1}\left(\|u\|_{L^{q\left(\mathbb{R}^{n}\right)}}^{q}\right) \geq C\left(\|u\|_{L^{q\left(\mathbb{R}^{n}\right)}}^{q}\right)^{\frac{p+q-2}{q^{\theta}}} .
$$

Then, from (3.2), we obtain

$$
\partial_{t}\|u(\cdot, t)\|_{L^{q\left(\mathbb{R}^{n}\right)}}^{q} \leq-C E(u) \leq-C H^{-1}\left(\|u\|_{L^{q\left(\mathbb{R}^{n}\right)}}^{q}\right) \leq-C\left(\|u\|_{L^{q\left(\mathbb{R}^{n}\right)}}^{q}\right)^{\frac{p+q-2}{q \theta}}
$$

from which it follows that

$$
\|u(\cdot, t)\|_{L^{q\left(\mathbb{R}^{n}\right)}}^{q} \leq C t^{-\frac{q \theta}{p+q-2-q \theta}}
$$

that is

$$
\|u(\cdot, t)\|_{L^{q}\left(\mathbb{R}^{n}\right)}^{q} \leq C t^{-\frac{(q-1) n}{q((p-2) n+2 \sigma)}},
$$

as we wanted to show.

Now we deal with the case $1<q<2$. We can interpolate, obtaining

$$
\|u(\cdot, t)\|_{L^{q\left(\mathbb{R}^{n}\right)}}^{q} \leq\|u(\cdot, t)\|_{L^{2}\left(\mathbb{R}^{n}\right)}^{q \theta}\|u(\cdot, t)\|_{L^{1}\left(\mathbb{R}^{n}\right)}^{q(1-\theta)},
$$

with

$$
\frac{1}{q}=\frac{\theta}{2}+(1-\theta), \quad \text { that is, } \theta=2\left(1-\frac{1}{q}\right) .
$$


As we have already proved that

$$
\|u(\cdot, t)\|_{L^{2}\left(\mathbb{R}^{n}\right)} \leq C t^{-\frac{n}{(p-2) n+2 \sigma}} \frac{1}{2} \text { and }\|u(\cdot, t)\|_{L^{1}\left(\mathbb{R}^{n}\right)} \leq C,
$$

we conclude

$$
\|u(\cdot, t)\|_{L^{q}\left(\mathbb{R}^{n)}\right.} \leq C t^{-\frac{n}{(p-2) n+2 \sigma}\left(1-\frac{1}{q}\right)}
$$

for $1<q<2$.

\section{Competing interests}

The authors declare that they have no competing interests.

\section{Authors' contributions}

All authors contributed equally to the writing of this paper. All authors read and approved the final manuscript.

\section{Author details}

${ }^{1}$ Departamento de Análisis Matemático, Universidad de Alicante, Ap. correos 99, Alicante, 03080, Spain. ${ }^{2}$ Departamento de Matemática, FCEyN UBA, Ciudad Universitaria, Pab 1, Buenos Aires, 1428, Argentina.

\section{Acknowledgements}

This work was partially supported by MEC MTM2010-18128 and MTM2011-27998 (Spain).

Received: 11 March 2014 Accepted: 25 April 2014 Published: 13 May 2014

\section{References}

1. Andreu-Vaillo, F, Mazon, JM, Rossi, JD, Toledo-Melero, JJ: Nonlocal Diffusion Problems. Mathematical Surveys and Monographs, vol. 165. Am. Math. Soc., Providence (2010)

2. Fife, P: Some nonclassical trends in parabolic and parabolic-like evolutions. In: Trends in Nonlinear Analysis, pp. 153-191. Springer, Berlin (2003)

3. Du, Q, Gunzburger, M, Lehoucq, R, Zhou, K: A nonlocal vector calculus, nonlocal volume-constrained problems, and nonlocal balance laws. Preprint

4. Bates, P, Chen, X, Chmaj, A: Heteroclinic solutions of a van der Waals model with indefinite nonlocal interactions. Calc. Var. 24, 261-281 (2005)

5. Hutson, V, Martínez, S, Mischaikow, K, Vickers, GT: The evolution of dispersal. J. Math. Biol. 47, 483-517 (2003)

6. Parks, ML, Lehoucq, R, Plimpton, S, Silling, S: Implementing peridynamics within a molecular dynamics code. Comput. Phys. Commun. 179, 777-783 (2008)

7. Silling, SA: Reformulation of elasticity theory for discontinuities and long-range forces. J. Mech. Phys. Solids 48 , 175-209 (2000)

8. Silling, SA, Lehoucq, R: Convergence of peridynamics to classical elasticity theory. J. Elast. 93, 13-37 (2008)

9. Ignat, L, Rossi, JD: Decay estimates for nonlocal problems via energy methods. J. Math. Pures Appl. (9) 92(2), 163-187 (2009)

10. Chasseigne, E, Chaves, M, Rossi, JD: Asymptotic behavior for nonlocal diffusion equations. J. Math. Pures Appl. (9) 86(3), 271-291 (2006)

11. Chasseigne, E, Felmer, P, Rossi, JD, Topp, E: Fractional decay bounds for nonlocal zero order heat equations. Preprint

12. Brändle, C, de Pablo, A: Nonlocal heat equations: decay estimates and Nash inequalities. Preprint (2014). arXiv:1312.4661v3

13. Ignat, L, Rossi, JD, San Antolin, A: Lower and upper bounds for the first eigenvalue of nonlocal diffusion problems in the whole space. J. Differ. Equ. 252(12), 6429-6447 (2012)

14. Ignat, L, Pinasco, D, Rossi, JD, San Antolin, A: Decay estimates for nonlinear nonlocal diffusion problems in the whole space. J. Anal. Math. 122(1), 375-401 (2014)

15. Di Neza, E, Palatucci, G, Valdinoci, E: Hitchhiker's guide to the fractional Sobolev spaces. Bull. Sci. Math. 136(5), 521-573 (2012)

10.1186/1687-2770-2014-109

Cite this article as: Esteve et al.: Upper bounds for the decay rate in a nonlocal $p$-Laplacian evolution problem. Boundary Value Problems 2014, 2014:109 